\title{
Erratum to: Regulation of IL12B Expression in Human Macrophages by TALEN-mediated Epigenome Editing
}

\author{
Meng CHEN ${ }^{1,2}$, Hua ZHU ${ }^{1,2}$, Yu-juan $\mathrm{MAO}^{1,2}$, Nan $\mathrm{CAO}^{1,2}$, Ya-li YU ${ }^{1,2}$, Lian-yun $\mathrm{LI}^{3}$, Qiu ZHAO ${ }^{1,2}$, Min WU $\mathrm{WU}^{3}$, Mei YE $\mathrm{YE}^{1,2 \#}$ \\ ${ }^{1}$ Department of Gastroenterology, Zhongnan Hospital, Wuhan University, Wuhan 430071, China \\ ${ }^{2}$ Hubei Clinical Center and Key Lab of Intestinal and Colorectal Diseases, Wuhan 430071, China \\ ${ }^{3}$ College of Life Sciences, Wuhan University, Wuhan 430072, China
}

(C) Huazhong University of Science and Technology 2020

\author{
Current Medical Science \\ https://link.springer.com/article/10.1007/s11596-020-2249-2 \\ 40(5):900-909,202
}

Prof. Mei YE works in the Department of Gastroenterology, Zhongnan Hospital, Wuhan University, and Hubei Clinical Center and Key Lab of Intestinal and Colorectal Diseases, as shown above. Her affiliation was incorrectly indicated in the article. 